\title{
Intrinsic Ion Selectivity of Narrow Hydrophobic Pores
}

\author{
Chen Song and Ben Corry* \\ School of Biomedical, Biomolecular and Chemical Sciences, The University of Western Australia, \\ Crawley, WA 6009, Australia
}

Received: November 17, 2008; Revised Manuscript Received: April 9, 2009

\begin{abstract}
We show that narrow hydrophobic pores have an intrinsic ion selectivity by using single-walled carbon nanotube membranes as a model. We examined pores of radius 3.4-6.1 $\AA$, and conducted molecular dynamics simulations to show that $\mathrm{Na}^{+}, \mathrm{K}^{+}$, and $\mathrm{Cl}^{-}$face different free energy barriers when entering hydrophobic pores. Most of the differences result from the different dehydration energies of the ions; however, changes in the solvation shell structure in the confined nanotube interior and van der Waals interactions in the small tubes can both play a role. Molecular dynamics simulations conducted under hydrostatic pressure show that carbon nanotube membranes can act as ion sieves, with the pore radius and pressure determining which ions will permeate through the membrane. This work suggests that the intrinsic ion selectivity of biological pores of differing radii might also play a role in determining their selectivity, in addition to the more common explanations based on electrostatic effects. In addition, "hydrophobic gating" can arise in continuous waterfilled pores.
\end{abstract}

\section{Introduction}

Recent studies have suggested that hydrophobic regions of membrane channel proteins can play an important role in controlling the transport of ions, water, and other solutes. Crystal structures have shown that many biological ion channels, including ligand-gated acetylcholine receptors, ${ }^{1}$ bacterial mechanosensitive channels, ${ }^{2,3}$ and potassium channels, ${ }^{4,5}$ contain long regions of their pores lined by nonpolar groups. It has been proposed that a "hydrophobic" mechanism can explain the gating of acetylcholine receptors that are not physically occluded in their closed state. ${ }^{6-8}$ It has also been proposed that hydrophobic effects can partly determine the selectivity of aquaporins. ${ }^{9}$ Thus, it would be very interesting to detail the ways in which hydrophobic pore linings can interact with ions and water molecules in channel proteins to ascertain the conditions under which these effects are important. Such an understanding can help to determine, for example, whether the known cation selectivity of acetylcholine receptors requires the presence of charged or polar groups to assist in cation transport or whether this can arise simply from the hydrophobic nature of part of the pore. Also, does the hydrophobic gating mechanism proposed for acetylcholine receptors require complete evacuation of water as seen in some simulations, or can it still work with a continuous water chain? Protein channels are generally complex in nature, and it is difficult to discriminate between the different factors that can influence gating and selectivity. Thus, a more efficient method for examining the role of hydrophobic regions of pores is to study simpler models in which various parameters can be controlled more easily. Many such model systems have been utilized, ${ }^{10-16}$ one of which is single-walled carbon nanotubes (SWNTs).

Carbon nanotubes have attracted much attention since their discovery, ${ }^{17}$ because of their many special structural, mechanical, and electronic properties. Many investigations have been carried

* Corresponding author. Address: School of Biomedical, Biomolecular and Chemical Sciences, University of Western Australia, Crawley, WA 6009, Australia. Tel.: +61 8 64883166. Fax: +61 8 64881005. E-mail: ben.corry@uwa.edu.au. out to study the mass-transport behavior of small molecules in carbon nanotubes, with particular attention being paid to the distribution and transport of water. ${ }^{18-25}$ Theoretical studies have shown that water molecules confined in narrow SWNTs exhibit distribution and diffusion properties distinct from those in the bulk and that these properties are tunable by changes of surface charge or deformation of the tube. ${ }^{26,27}$ Studies on gases, ${ }^{28}$ protons, ${ }^{29,30}$ and ion transport ${ }^{31-33}$ in SWNTs have also been performed. Most of these studies have shown that the transport is much faster than that in other narrow channels, which is probably due to the smoothness of the interior of SWNTs. Therefore, SWNTs can be an efficient mass-transport medium in nanotechnology. In addition, it has been shown that ions and water molecules face different free energy barriers when passing through membranes composed of SWNTs, which suggests a very practical application in desalination. ${ }^{25}$ At the same time, experimental techniques have made it possible to fabricate various nanotube-based channels and membranes, ${ }^{23,33-35}$ with controllable pore diameters and lengths. ${ }^{36,37}$ Therefore, studying the interactions between small species and SWNT membranes is interesting and necessary for the potential application of these materials in nanomembrane technology.

Motivated both by our interest in understanding biological channels and by the technological applications, we extend previous work by examining how a range of different ions interact with and permeate through SWNT membranes with different pore radii using molecular dynamics (MD) simulations. Specifically, we used the hexagonally packed $(5,5),(6,6),(7,7)$, $(8,8)$, and $(9,9)$ SWNTs as model hydrophobic nanoporous membranes and calculated the potentials of mean force (PMF) for $\mathrm{Na}^{+}, \mathrm{K}^{+}$, and $\mathrm{Cl}^{-}$passing through the pores. Unlike the previous work of Lynden-Bell and Rasaiah, which focused on the diffusion of ions and water in an infinite cylinder, ${ }^{10}$ we are more interested in the process of ions entering the pores from bulk water, which is believed to be the key process that determines the free energy barriers and thus the intrinsic ion selectivity of pores. Peter and Hummer have studied the PMF and spontaneous transport of the $\mathrm{Na}^{+}$in $(10,10)$ SWNTs. ${ }^{12}$ 


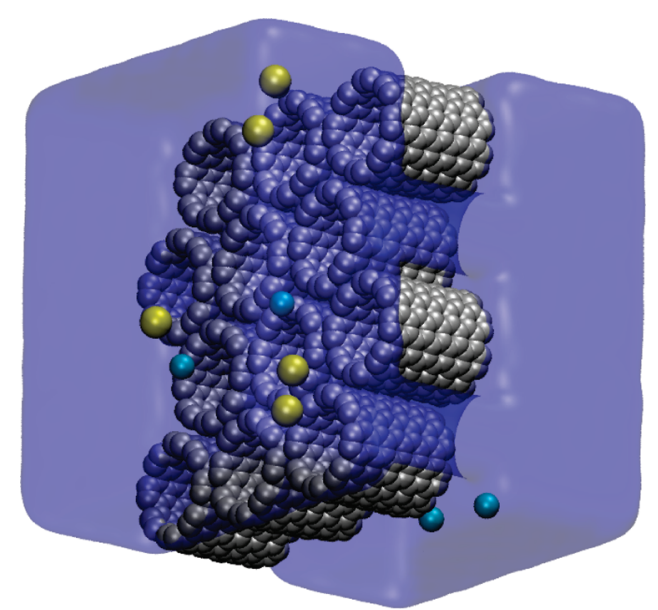

Figure 1. One unit cell of the SWNT membrane model system, which is composed of 12 hexagonally packed SWNTs immersed in a solution of $\mathrm{NaCl}$ in water.

Although they noted that $\mathrm{Na}^{+}$can pass whereas $\mathrm{Cl}^{-}$cannot, they provided no further discussion. Liu et al. studied ion selectivity in $(10,0)$ and $(11,0)$ SWNTs, found that these SWNTs favor the passage of $\mathrm{K}^{+}$over $\mathrm{Na}^{+}$, and investigated the conditions that allow $\mathrm{Na}^{+}$to pass by controlling the temperature and pressure, ${ }^{38}$ but they performed no quantitative analysis of the origin of this selectivity. An extensive study of the ion selectivity of hydrophobic pores with various radii is thus still absent. Therefore, in the present work, we focus on the origin of the ion selectivity of the pores, noting that selectivity can arise between similar ions such as $\mathrm{Na}^{+}$and $\mathrm{K}^{+}$and that the selectivity sequence can change depending on the radius of the nanotubes. Because the hydrophobic gating hypothesis for ion channels ascribes the gating of the pore to the dehydration energy of the permeant ions, we explicitly determine the dehydration free energy of each ion for comparison with the energy barrier seen upon entering the nanotubes, to establish its contribution to the ion selectivity. Simulations were also carried out under hydrostatic pressure to explicitly model the dynamic transport of ions and water. These results show that narrow hydrophobic pores have an intrinsic ion selectivity, which might assist in understanding selectivity in biological pores. These results also suggest a potential application of carbon nanotubes as ion sieves for use in nanotechnology.

\section{Methods}

Model System. Hydrophobic porous membranes were created by hexagonally packing 12 SWNTs each $\sim 13.5$ - $\AA$-long following previous models, ${ }^{16,25}$ as shown in Figure 1. These were solvated by $\sim 20$ - $\AA$-thick layers of water on either side, with ions randomly placed to yield a net concentration of $250 \mathrm{mM}$ $\mathrm{NaCl}$ or $\mathrm{KCl}$ in one unit cell. A continuous two-dimensional membrane was created by using periodic boundary conditions. Such model systems were constructed for $(5,5),(6,6),(7,7)$, $(8,8)$, and $(9,9)$ SWNTs with radii of $3.39,4.07,4.75,5.42$, and $6.10 \AA$, respectively. In our simulations, all of the SWNT axes were parallel to the $z$ axis, and all of the tubes were located between $z=-7.0$ and $z=7.0 \AA$, with the center at $z=0 \AA$.

MD Simulations. All MD simulations were performed using the NAMD2.6 package $^{39}$ with the CHARMM27 force field. ${ }^{40}$ The parameters of all SWNT carbon atoms were set to be those of $\mathrm{sp}^{2}$-like aromatic carbons (type CA) in the CHARMM27 force field, including bond, angle, and dihedral parameters. All charges of these carbon atoms were set to 0 . The choice of these parameters has been validated by previous simulations. ${ }^{16,25}$ The TIP3P water model was employed. Before data collection, all systems were energy minimized and equilibrated for $3 \mathrm{~ns}$ under a constant pressure of $1 \mathrm{~atm}$ and a temperature of $300 \mathrm{~K}$, during which time a harmonic constraint applied to the carbon atoms was gradually reduced from 2 to $0.1 \mathrm{kcal} /(\mathrm{mol} \AA)$. Apart from the simulations conducted with pressure differences discussed below, harmonic constraints of $0.1 \mathrm{kcal} /(\mathrm{mol} \AA)$ were applied to all carbon atoms during all of the simulations to keep the membrane roughly in place. The time step was set to $1 \mathrm{fs}$ for all simulations. Langevin dynamics was used to control the temperature and pressure. A value of $12 \AA$ was used to cut off the nonbonded van der Waals interactions, and the particle mesh Ewald (PME) method was used to calculate the full electrostatic interactions. Coordination numbers were determined by the average number of ligands within the distance of the first minimum in the radial distribution function.

PMF Calculations. The potentials of mean force (PMF) for ions passing through one of the SWNTs were determined using umbrella sampling ${ }^{41}$ in which the harmonic biasing potential

$$
\frac{k_{r}}{2}\left(r-r_{i}\right)^{2}+\frac{k_{z}}{2}\left(z-z_{i}\right)^{2}
$$

was applied to the test ion, where $r$ and $z$ are the radial and axial coordinates, respectively, of the ion defined from the center of one of the pores; $r_{i}$ and $z_{i}$ are target ion positions; and $k_{r}$ and $k_{z}$ are corresponding force constants. The target positions were moved from $z=17$ to $z=-2 \AA$ along the tube axis $\left(r_{i}=0\right)$ using the force constant $k_{z}=2.0 \mathrm{kcal} /\left(\mathrm{mol} \AA^{2}\right) . k_{r}$ was set to 0.1 for the $(7,7),(8,8)$, and $(9,9)$ SWNTs and to 0.2 for the $(5,5)$ and $(6,6)$ SWNTs. The sampling window width along the $z$ axis was set to $1 \AA$ for the $(7,7),(8,8)$, and $(9,9)$ SWNT systems, whereas for the $(5,5)$ and $(6,6)$ SWNTs, the width was set to $0.5 \AA$. Simulations using larger force constants $\left[k_{z}=9.0\right.$ $\left.\mathrm{kcal} /\left(\mathrm{mol} \AA^{2}\right)\right]$ were used to better sample the entrance of the narrow $(5,5)$ and $(6,6)$ SWNTs where the forces are large. Additional windows with target positions at $r=5 \AA$ in the bulk water and $r=1 \AA$ in the tube interior were utilized to create two-dimensional PMF profiles. For each window, a 500-ps simulation was run, and the coordinate of the test ion was written every 5 fs. The first 100 ps of each run was discarded as equilibration. Collective analysis of the data was performed using the weighted histogram analysis method ${ }^{42,43}$ according to the implementation of Grossfield ${ }^{44}$ to get the one-dimensional (1D) or two-dimensional (2D) PMF, with 0.00001 as the convergence criteria and 100 bins along the $z$ axis to generate the 1D PMF profiles or 40 bins along the $r$ axis for 2D PMF profiles. All of the PMF profiles should be considered one-ion PMF profiles as no other ions enter the pores during the simulations. Altogether, $330 \mathrm{~ns}$ of MD simulations were performed to obtain the PMF profiles for all of the types of ions and SWNT membranes.

Numerous tests were made to check the accuracy of our PMF calculations. First, PMF profiles were obtained from the first and second halves of the data, which agreed with each other and with the PMF from all of the data to within $0.5 \mathrm{kcal} / \mathrm{mol}$ in all cases. A PMF was also obtained for the entire length of the $(8,8)$ nanotube; it was found to be symmetric, with almost no difference between the energies on the left and right sides of the pore. Finally, the data presented are consistent with additional simulations made for some cases using twice as many umbrella windows and a longer sampling time (1 ns per window).

FEP Calculations. To calculate the dehydration energies of the ions, we used the method of alchemical free energy 


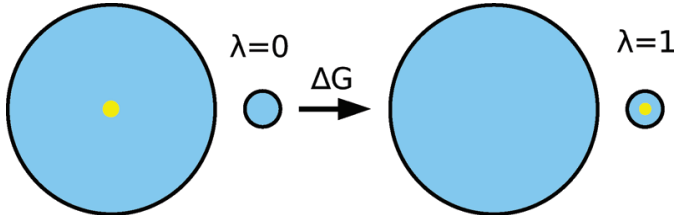

Figure 2. Sketch of the free energy perturbation calculations. The blue spheres represent the water spheres, and the yellow points represent the test ion. $\lambda=0$ indicates the initial state in which the test ion is located at the center of the bulk-water sphere, and $\lambda=1$ indicates the final state in which the test ion has switched its position with a water molecule at the origin of the small sphere.

perturbation (FEP). ${ }^{45}$ To create a dehydration scenario similar to that undergone by ions entering the nanotubes, we first generated a water sphere of radius $15 \AA$ with the test ion fixed at the center to represent the bulk solution, taken from a wellequilibrated bulk water solution. Placed beside the large sphere was another smaller water sphere that contained the desired number of water molecules and had a radius equal to that of the first water shell of the test ion $\left(3.2 \AA\right.$ for $\mathrm{Na}^{+}, 3.85 \AA$ for $\mathrm{Cl}^{-}$and $3.6 \AA$ for $\mathrm{K}^{+}$). The oxygen atom of one water molecule was fixed at the center of the small sphere, whereas all of the other water molecules were free to move within it. The distance between the center of the two spheres was $20 \AA$ to mimic the dehydration process in SWNTs. High constraints [100 $\left.\mathrm{kcal} /\left(\mathrm{mol} \AA^{2}\right)\right]$ were applied to the two spheres at the sphere boundaries to keep all of the atoms in the constant-volume spheres, as was done previously by Thomas et al. ${ }^{46}$ Then, as sketched in Figure 2, alchemical FEP calculations were performed for these systems in which the test ion was moved from the bulk-water sphere to the small water cluster, replacing a water molecule that was moved in the opposite direction, mimicking the dehydration processes encountered by ions entering the nanopores. The free energy change, $\Delta G$, was determined as

$$
\text { ion/(bulk) } \mathrm{H}_{2} \mathrm{O}+(n+1) \mathrm{H}_{2} \mathrm{O} \stackrel{\Delta G}{\longrightarrow}\left(\text { bulk }+\underset{\text { ion } / n \mathrm{H}_{2} \mathrm{O}}{1} \mathrm{H}_{2} \mathrm{O} \underset{+}{+}\right.
$$

where $n$ is the number of water molecules in the small sphere after the calculation, which ranged from 0 to 6 for $\mathrm{Na}^{+}$, from 0 to 8 for $\mathrm{Cl}^{-}$, and from 0 to 7 for $\mathrm{K}^{+}$. The FEP calculations employed the so-called dual-topology approach. ${ }^{47,48}$ During the simulations, the ion was morphed from one sphere to the other in unequally spaced steps, with smaller windows at each end of the process to improve sampling. The parameter $\lambda$ was used to measure progress along the alchemical calculation, with $\lambda=$ 0 indicating the initial state and $\lambda=1$ indicating the final state (see Figure 2). The step size, $\Delta \lambda$, was 0.05 in the range from 0.1 to 0.9 , and $\lambda$ values of $(0,0.0000001,0.000001,0.00001$, $0.0001,0.01,0.05,0.1)$ and $(0.9,0.95,0.99,0.999,0.9999$, $0.99999,0.999999,1)$ were used to sample the end windows. For each window, a 100-ps simulation was run, and the first 50 ps was considered as equilibration. In total, more than $170 \mathrm{~ns}$ of FEP MD simulations was performed to calculate the dehydration energies. Because periodic boundary conditions could not be employed for this situation, long-range interactions were all cut off at $12 \AA$. It should be noted here that changes in the distance between the two spheres, the radius of the large sphere, and the cutoff value of long-range interactions can all affect the final results as described in the Results and Discussion section. The values used in this study were chosen to represent the dehydration process in this specific case. (See the Supporting Information for more details.) To assess the uncertainties in the
FEP data, a total of seven independent trajectories were calculated with different random starting velocities for six cases, those for each ion type with either zero or the maximum number of water molecules in the first solvation shell. The uncertainty in each case was estimated from the standard deviation in the dehydration energies obtained from the seven simulations.

Hydrostatic-Pressure Simulations. The method developed by Zhu et al. ${ }^{49,50}$ was employed to introduce a hydrostatic pressure difference in our simulations, as described previously. ${ }^{25}$ Briefly, for each nanotube type, a constant force was applied to water molecules greater than $13 \AA$ from either side of the membrane, to generate a hydrostatic pressure difference across the membrane. The simulation systems were similar to those in the PMF calculations, except that both $\mathrm{NaCl}$ and $\mathrm{KCl}$ were included in the solution, resulting $250 \mathrm{mM} \mathrm{Na}^{+}, 250 \mathrm{mM} \mathrm{K}^{+}$, and $500 \mathrm{mM} \mathrm{Cl}^{-}$. To keep the membrane in place and avoid overall translation of the system, harmonic constraints of 0.8 $\mathrm{kcal} /\left(\mathrm{mol} \AA^{2}\right)$ were applied to all carbon atoms. For each of the five membranes, 20-ns hydrostatic-pressure MD simulations were performed under pressures of 100 and $200 \mathrm{MPa}$. The total simulation time was $200 \mathrm{~ns}$.

\section{Results and Discussion}

Our previous study showed that water molecules and $\mathrm{Na}^{+}$ face different free energy barriers when entering SWNT pores. ${ }^{25}$ In that study, water molecules spontaneously entered all of the nanotubes and moved easily through them. However, $\mathrm{Na}^{+}$was shown to have a pore-size-dependent energy barrier that made entering narrow pores difficult. In this study, we repeated those calculations using a more rigid SWNT model; specifically, in addition to the bond and angle terms, the dihedral term was also taken into account during calculations of the energies of the SWNTs in the CHARMM force field. Results similar to those in the previous study were obtained: In the equilibrating simulations, water entered all of the pores (in bursts in the narrowest pore) as a result of the tight hydrogen-bonding network between adjacent water molecules, but no ions entered because they faced larger barriers than water molecules. As expected, the barriers for the corresponding cases were higher than the previous results because of the greater rigidity of the pores. Surprisingly, water molecules were found to encounter the smallest energy barrier when entering the narrowest $(5,5)$ pore, as can be seen in Figure 3a. The PMF for water moving along the tube axis exhibits a wavelike curve, which means that the distribution of water molecules inside the $(5,5)$ SWNT is steplike rather than continuous, with some positions favored over others, as shown in Figure 4. This result is consistent with the previous work done by Hummer et al., ${ }^{18}$ in which a similar water distribution and transmission behavior were found.

In contrast to the water molecules, which spontaneously entered all of the pores studied, ions were found to face a distinct free energy barrier upon entering the pores, as shown in the 1D PMF profiles in Figure $3 b-f$. As it is thought that the dehydration effect is the origin of the free energy barrier (discussed in more detail below), an interesting question is whether narrow hydrophobic pores have intrinsic ion selectivities, given that different ions have different dehydration energies. The results of our PMF calculations show that they do, and notably, that the order of selectivity is not the same in each of the pores. As shown in Figure $3 b-f$, different ions face different free energy barriers when entering pores of a particular radius. For example, in the $(5,5)$ tube, the order of free energy barriers upon entry is $\Delta G_{\mathrm{Cl}^{-}}>\Delta G_{\mathrm{Na}^{+}}>$ $\Delta G_{\mathrm{K}^{+}}$, which means that it is easiest for $\mathrm{K}^{+}$to enter the $(5,5)$ 

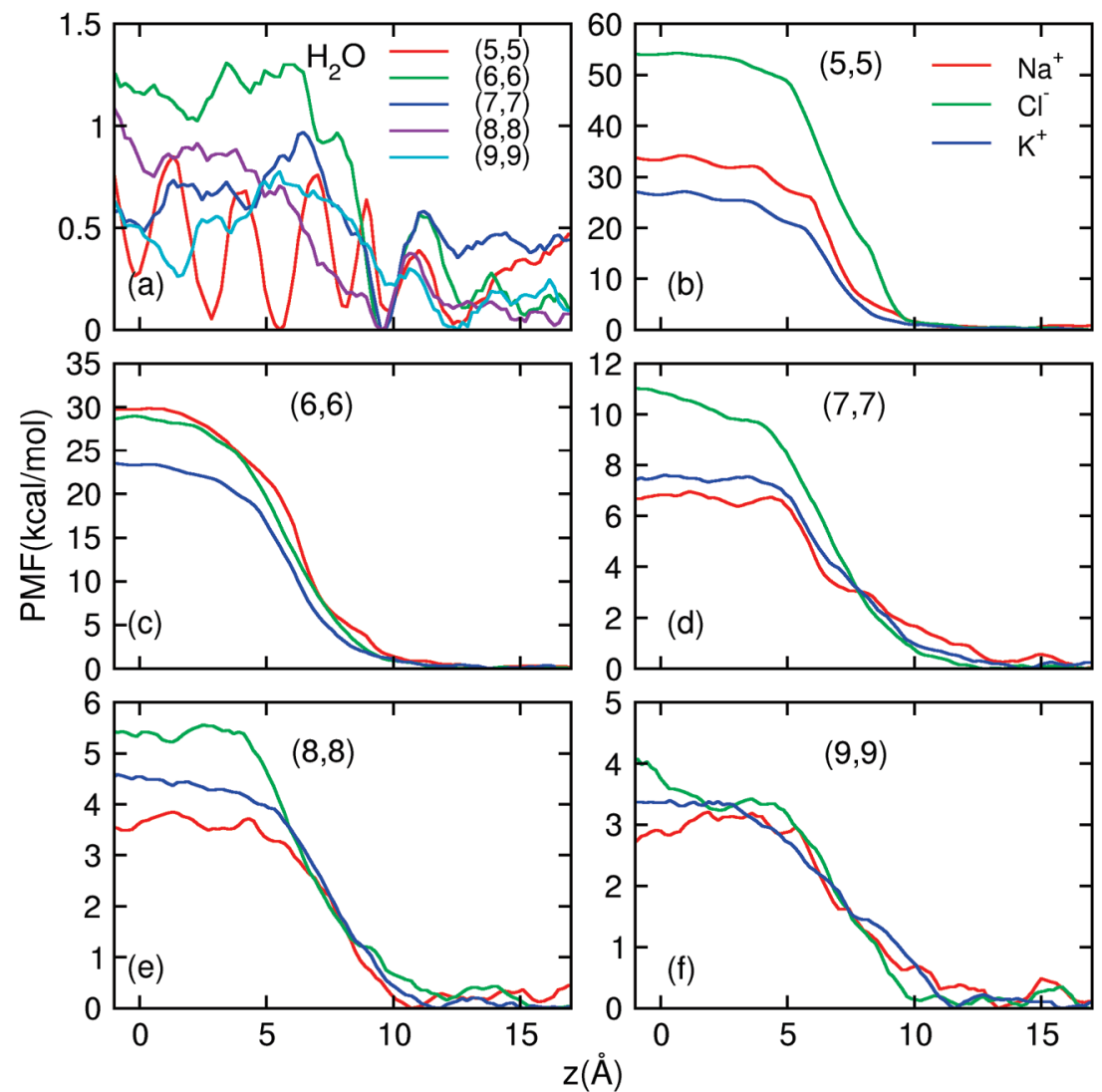

Figure 3. PMF profiles of a water molecule or ions within the carbon nanotubes. (a) PMF for water molecules in the five types of SWNTs studied. $(b-f)$ PMF profiles for the three types of test ions in $(5,5),(6,6),(7,7),(8,8)$, and $(9,9)$ nanotubes, respectively. The centers of SWNTs are located at $z=0.0$, and the tubes end at around $z=7.0$.

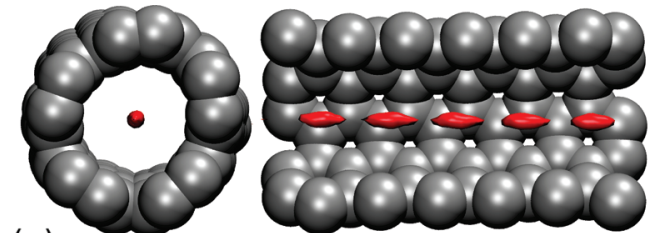

(a)

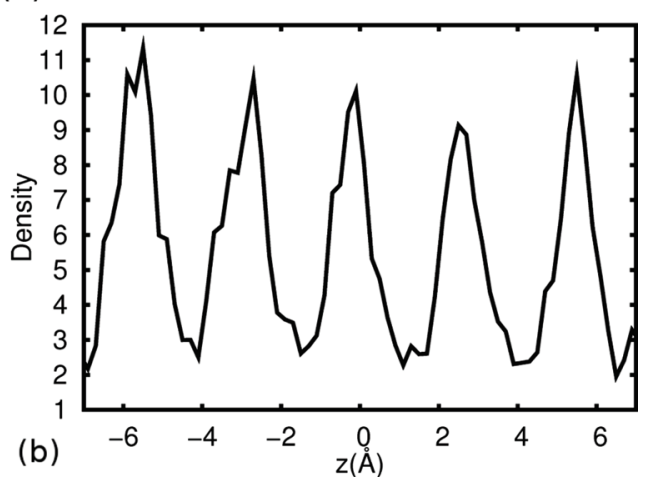

Figure 4. Steplike distribution of water inside a $(5,5)$ SWNT. (a) Top and side views of the water distribution inside $(5,5)$ SWNT. The red isosurface was plotted according to the average oxygen density. For clarity, half of the SWNT was removed in the side view. (b) Average oxygen density along the tube axis.

SWNT. In contrast, for the $(6,6)$ SWNT, $\Delta G_{\mathrm{Na}^{+}} \approx \Delta G_{\mathrm{Cl}^{-}}>$ $\Delta G_{\mathrm{K}^{+}}$, where the discrimination between $\mathrm{Na}^{+}$and $\mathrm{Cl}^{-}$is not as obvious as for the $(5,5)$ SWNT. These results are consistent with the study of Liu et al., ${ }^{31}$ who observed $\mathrm{K}^{+}$selectivity in a $(10,0)$ SWNT whose diameter $(7.83 \AA)$ lies between that of the $(5,5)$ and $(6,6)$ SWNTs described here. For the $(7,7)$ and $(8,8)$ SWNTs, $\Delta G_{\mathrm{Cl}^{-}}>\Delta G_{\mathrm{K}^{+}} \geq \Delta G_{\mathrm{Na}^{+}}$, whereas for the
$(9,9)$ SWNTs, the PMF barriers are no longer distinguishable. Although the 1D PMF profiles provide the simplest visual representation of the barriers to ion permeation, it has been noted that a 2D PMF profiles provide a more reliable picture of the free energy of the particle relative to bulk. ${ }^{51}$ For this reason, we calculated $2 \mathrm{D}$ PMF profiles for each ion to traverse the pores, examples of which are shown in Figure 5. The corresponding energy barriers faced by ions passing through the pores in the 1D and 2D PMF profiles are listed in Table 1 and yield similar barriers heights and selectivity sequences. It can be seen from the $2 \mathrm{D}$ PMF profiles that $\mathrm{K}^{+}$ prefers to reside on the central axis of the pores, but that $\mathrm{Na}^{+}$does not.

As it is easier for some types of ions to pass through some of the pores than others, it is clear that the size of the hydrophobic pore in a channel protein can play a role in determining the selectivity. This effect could act in addition to the local electrostatic environment that is usually ascribed as the origin of selectivity in biological pores ${ }^{52}$ From the viewpoint of nanotechnology, it is also possible to discriminate different types of ions by using SWNT-composed membranes, as discussed in detail below. It should be noted that there are very high free energy barriers for ions to permeate through $(5,5)$ and $(6,6)$ SWNTs, so no ion permeation could be expected under biologically relevant driving forces. $(9,9)$ SWNTs have relatively low ion selectivities because of the small free energy differences between types of ions. However, for $(7,7)$ or $(8,8)$ SWNTs, the free energy differences between different types of ions are $\sim 2$ $\mathrm{kcal} / \mathrm{mol}$, which are biologically relevant values and are not negligible. Previous studies have shown that the free energy difference leading to a 1000 -fold selectivity for $\mathrm{K}^{+}$over $\mathrm{Na}^{+}$ 

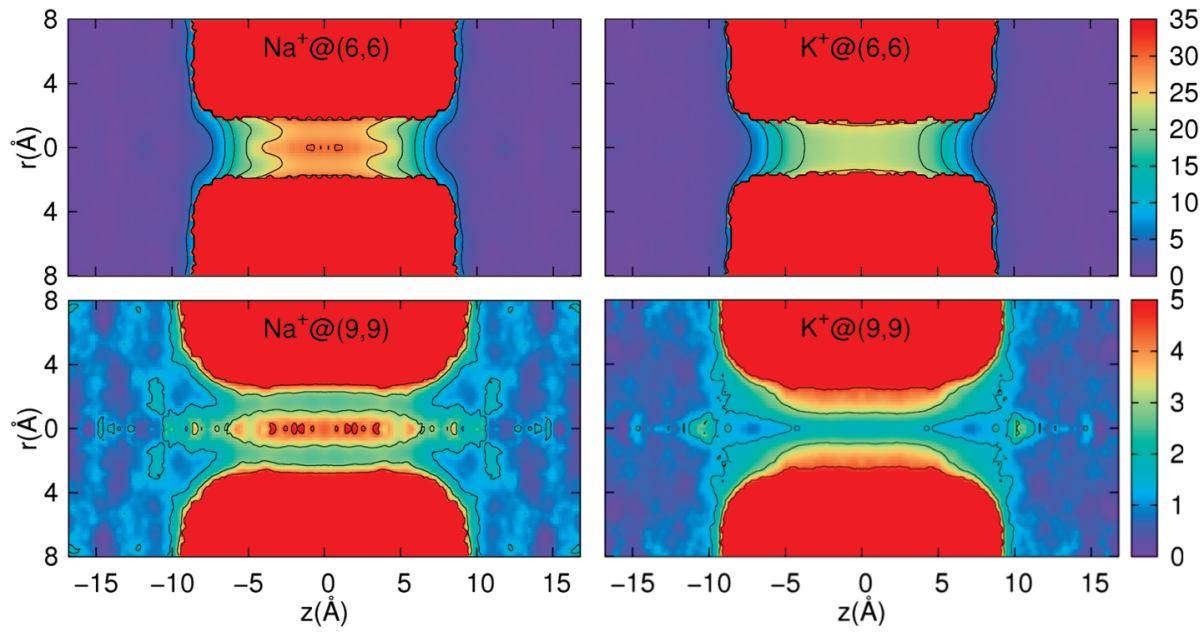

Figure 5. Two-dimensional PMF profiles for $\mathrm{Na}^{+}$and $\mathrm{K}^{+}$in $(6,6)$ and $(9,9) \mathrm{SWNT}$ systems.

in potassium channels, for example, is less than $\sim 6 \mathrm{kcal} / \mathrm{mol} .{ }^{46}$ A $2 \mathrm{kcal} / \mathrm{mol}$ energy difference should similarly produce a 10 fold difference in ion permeation. These results highlight the potential importance of the unrecognized intrinsic hydrophobic ion selectivity of biological pores that could complement the well-known electrostatic origins of ion selectivity. ${ }^{52}$

To investigate whether the free energy barriers observed for ions entering nanotubes can be ascribed to the energy required to (partially) dehydrate the ions, we first analyzed the coordination numbers of the test ions when they entered each of the pores during umbrella sampling simulations. As shown in Figure 6 , the coordination numbers for $\mathrm{Na}^{+}, \mathrm{Cl}^{-}$, and $\mathrm{K}^{+}$in bulk water are about 6,8 , and 7 , respectively. To enter the narrower tubes, however, the ions must be stripped of some of this water. It is only in the widest tube studied that the coordination number in the pore can approximate that in the bulk.

To further isolate the effects of partial dehydration from other influences, we estimated the dehydration energy for each ion type to leave the bulk and be coordinated by only a few water molecules as summarized in Table 2. As expected, the fewer water molecules remaining around the ion, the larger the free energy required to remove it from bulk. Combining Figure 6 and Table 2 together, it is possible to estimate how much energy is needed to move an ion into each tube considering dehydration energy alone. For example, for a $\mathrm{Na}^{+}$ion to be placed into a $(5,5)$ SWNT, only two water molecules can be left around the ion in the SWNT, as can be seen in Figure 6a. From Table 2, we know that the free energy change from taking all of the other water molecules away from the $\mathrm{Na}^{+}$is about $33 \mathrm{kcal} /$ mol, which is close to the corresponding PMF barrier shown in Figure 3. In general, the dehydration energy can explain much of the trend in PMF barriers seen in Figure 3 and the swap in selectivity sequence in the pores. Considering just the results for $\mathrm{Na}^{+}$and $\mathrm{K}^{+}$, one can see that the narrowest pore enforces the same coordination number (2) for both ion types. Thus, $\mathrm{Na}^{+}$, with the larger dehydration energy faces a larger barrier for entry. In the wider pores, however, the smaller $\mathrm{Na}^{+}$is able to gain a coordination number that is larger or closer to its bulk

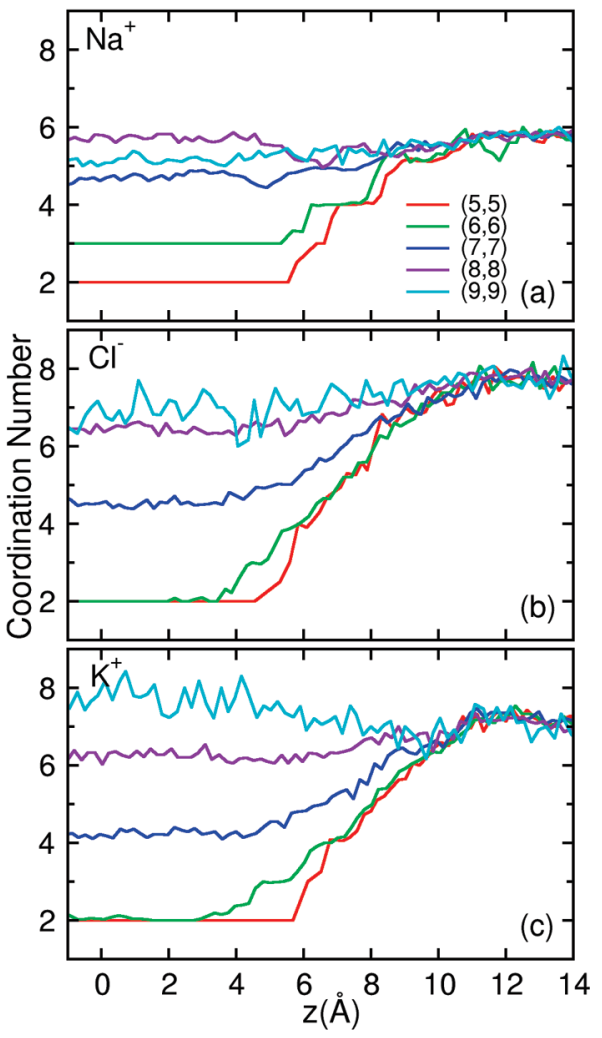

Figure 6. Coordination numbers of (a) $\mathrm{Na}^{+}$, (b) $\mathrm{Cl}^{-}$, and (c) $\mathrm{K}^{+}$ entering each of the carbon nanotubes studied. The pore center is at $z$ $=0.0$.

value, and thus, the dehydration energy is lower than for $\mathrm{K}^{+}$. We note that the dehydration energies calculated in our FEP simulations depend on some of the parameters chosen. In this case, the large and small spheres were kept fairly close together to represent dehydration of ions at the mouths of the pores. Moving the spheres farther apart takes the complete dehydration energy of the ions closer to the experimental dehydration

TABLE 1: Energy Barriers (kcal/mol) from One-Dimensional and Two-Dimensional PMF Profiles

\begin{tabular}{|c|c|c|c|c|c|c|c|c|c|c|}
\hline & \multicolumn{2}{|c|}{$(5,5)$} & \multicolumn{2}{|c|}{$(6,6)$} & \multicolumn{2}{|c|}{$(7,7)$} & \multicolumn{2}{|c|}{$(8,8)$} & \multicolumn{2}{|c|}{$(9,9)$} \\
\hline & $1 \mathrm{D}$ & $2 \mathrm{D}$ & $1 \mathrm{D}$ & $2 \mathrm{D}$ & $1 \mathrm{D}$ & $2 \mathrm{D}$ & $1 \mathrm{D}$ & $2 \mathrm{D}$ & $1 \mathrm{D}$ & $2 \mathrm{D}$ \\
\hline $\mathrm{Na}^{+}$ & 34.34 & 34.27 & 29.81 & 29.58 & 6.95 & 5.43 & 3.85 & 2.37 & 3.21 & 2.94 \\
\hline $\mathrm{Cl}^{-}$ & 54.32 & 52.02 & 28.97 & 27.61 & 11.04 & 10.23 & 5.56 & 5.06 & 4.07 & 3.97 \\
\hline
\end{tabular}


TABLE 2: Free Energy (kcal/mol) Required to Fully or Partly Dehydrate Each Type of Ion ${ }^{a}$

\begin{tabular}{|c|c|c|c|c|c|c|c|c|c|}
\hline & \multicolumn{9}{|c|}{$n$} \\
\hline & 0 & 1 & 2 & 3 & 4 & 5 & 6 & 7 & 8 \\
\hline $\mathrm{Na}^{+}$ & $74.80(0.14)$ & 50.26 & 33.47 & 21.39 & 12.63 & 7.15 & $4.96(0.60)$ & N/A & N/A \\
\hline $\mathrm{K}^{+}$ & $52.55(0.20)$ & 36.69 & 24.41 & 16.80 & 10.55 & 6.70 & 4.23 & $2.49(0.47)$ & N/A \\
\hline
\end{tabular}

${ }^{a}$ Each value represents the energy to remove an ion from the bulk to a situation in which it is surrounded by $n$ water molecules as determined from FEP calculations. The standard deviations from seven independent simulations are given in parentheses for $n=0$ and for the maximum number of water molecules in the first water shell.

TABLE 3: Differences in the Nonbonded Energies (kcal/ mol) for the Test Ion in the Bulk and in the Center of the Nanotube

\begin{tabular}{lccccc}
\hline \multicolumn{1}{c}{ size } & total & $\begin{array}{c}\text { neighboring } \\
\text { water }\end{array}$ & $\begin{array}{c}\text { other pore } \\
\text { water }\end{array}$ & nanotube & bulk \\
\hline$(5,5) \mathrm{Na}^{+}$ & 12.77 & 10.0 & -2.3 & -2.03 & 7.11 \\
$(6,6)$ & 8.36 & 7.83 & -3.74 & -1.7 & 5.96 \\
$(7,7)$ & 1.97 & 3.77 & -4.52 & -0.98 & 3.69 \\
$(8,8)$ & 1.14 & 0.78 & -5.33 & -0.58 & 6.27 \\
$(9,9) \mathrm{Na}^{+}$ & 0.61 & 2.27 & -7.71 & -0.58 & 6.64 \\
& & & & & \\
$(5,5) \mathrm{Cl}^{-}$ & 17.93 & 5.04 & -2.09 & 5.44 & 9.53 \\
$(6,6)$ & 2.95 & 5.09 & -1.05 & -7.14 & 6.04 \\
$(7,7)$ & 1.41 & 4.01 & -3.75 & -5.71 & 6.86 \\
$(8,8)$ & -1.42 & 2.57 & -5.4 & -4.45 & 5.86 \\
$(9,9) \mathrm{Cl}^{-}$ & 2.4 & 0.99 & -7.73 & -3.2 & 12.33 \\
& & & & & \\
$(5,5) \mathrm{K}^{+}$ & 9.99 & 6.94 & -1.04 & -3.53 & 7.62 \\
$(6,6)$ & 5.89 & 7.35 & -2.23 & -3.45 & 4.21 \\
$(7,7)$ & 3.17 & 4.47 & -3.77 & -2.61 & 5.09 \\
$(8,8)$ & 1.65 & 2.04 & -7.24 & -1.67 & 8.52 \\
$(9,9) \mathrm{K}^{+}$ & -0.43 & 1.16 & -7.99 & -1.02 & 7.42
\end{tabular}

enthalpies. The differences in the complete dehydration energies seen in our results are in almost perfect agreement with the differences in the experimental dehydration enthalpies, indicating that our values provide a good representation of dehydration for the cases shown.

There are, however, cases in which the dehydration energy alone does not adequately explain the size of the PMF barrier. The most obvious difference is between the PMF barrier and the corresponding dehydration energy for $\mathrm{Cl}^{-}$in the $(5,5)$ SWNT. Whereas the dehydration energy expected for it to enter this pore is about $28 \mathrm{kcal} / \mathrm{mol}$, the PMF barrier is about $57 \mathrm{kcal} /$ mol. For the $(6,6)$ pore, however, the dehydration energy for $\mathrm{Cl}^{-}$closely matches the PMF barrier. Obviously, the dehydration energy is not the only factor that influences the free energy of the ion entering this narrow pore. To determine which interactions might affect the PMF barrier, we decomposed the potential energy change between the ion and its surroundings upon entering the pores, which showed that, when $\mathrm{Cl}^{-}$enters the $(5,5)$ SWNT, there is a strong repulsive (van der Waals) interaction between the $\mathrm{Cl}^{-}$ion and the carbon nanotube, essentially because the ion is too large to fit into the pore. In contrast, the interactions between the ion and the nanotube are attractive in all other cases, as shown in Table 3. Therefore, the van der Waals interaction between the ion and the SWNT must also play a role in determining the free energy barrier, especially in the narrower SWNTs, which is consistent with the work of Tai et al. ${ }^{53}$

We also note that previous studies have shown that there are changes in the geometry of the first hydration shell of the ions upon narrow cylindrical confinement. ${ }^{10,31}$ This fact helps to to explain some unusual behavior in the coordination number shown in Figure 6. The $\mathrm{Na}^{+}$ion has about six coordinated water

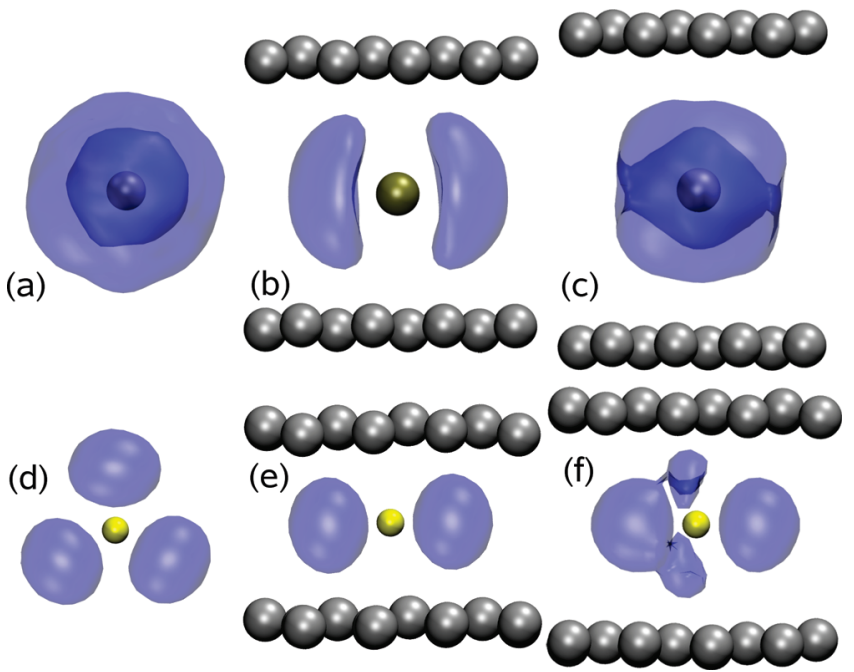

Figure 7. $(\mathrm{a}-\mathrm{c})$ First water shells around $\mathrm{K}^{+}$in the bulk, a $(7,7)$ SWNT, and a $(9,9)$ SWNT, respectively, represented by isosurface plots of the average water density. Light blue indicates the locations with higher water density, whereas dark blue indicates the vacuum between the $\mathrm{K}^{+}$ion and the first water shell. $(\mathrm{d}-\mathrm{f})$ First water shells around $\mathrm{Na}^{+}$in a small water sphere composed of three water molecules, in a $(5,5) \mathrm{SWNT}$, and in a $(6,6) \mathrm{SWNT}$, respectively. The tube axis points from left to right, with the ion and some carbon atoms on tube wall shown for clarity.

molecules inside the $(8,8) \mathrm{SWNT}$, whereas it has only about five inside the wider $(9,9)$ pore; $\mathrm{K}^{+}$inside the $(9,9)$ SWNT has a larger number of molecules in its first solvation shell than in the bulk! This behavior can be explained by the effects that confinement has on the solvation shell structure. Figure $7 a-c$ shows the first-shell water densities around $\mathrm{K}^{+}$in the bulk, in a $(7,7)$ SWNT, and in a $(9,9)$ SWNT, respectively. The first solvation shell of $\mathrm{K}^{+}$in the bulk is spherelike. In the $(7,7)$ SWNT (and also narrower SWNTs), the water molecules mainly distribute along the tube axis, avoiding being located between the ion and the tube wall. Interestingly, in the $(9,9)$ SWNT, the water molecules prefer to be located between the ion and the tube wall where they can form the greatest number of van der Waals interactions, forming a tubelike distribution with more water molecules coordinating with the $\mathrm{K}^{+}$ion than in the bulk. Another case in which the confinement alters the water geometry is for $\mathrm{Na}^{+}$in the $(6,6)$ SWNT, as shown in Figure 7d-f. Although the ion gains a coordination number of three in the $(6,6)$ SWNT, as shown in Figure 7f, the geometry of these water molecules is very different from that seen in the absence of confinement (Figure 7d). For this reason, the energy barrier to $\mathrm{Na}^{+}$in the $(6,6)$ tube lies between that expected for partial dehydrations to coordination numbers of 2 and 3 . As a consequence, $\mathrm{K}^{+}$faces a lower energy barrier to enter the pore, despite having a lower coordination number.

Because different ions face different PMF barriers to enter the SWNTs, it is expected that the SWNT membranes can act 
TABLE 4: Numbers of Ions and Water Molecules Passing Through the Membranes within 20 ns Under Different Pressures

\begin{tabular}{|c|c|c|c|c|c|c|c|c|}
\hline & \multicolumn{4}{|c|}{$200 \mathrm{MPa}$} & \multicolumn{4}{|c|}{$100 \mathrm{MPa}$} \\
\hline & $\overline{\mathrm{Na}^{+}}$ & $\mathrm{Cl}^{-}$ & $\mathrm{K}^{+}$ & $\mathrm{H}_{2} \mathrm{O}$ & $\overline{\mathrm{Na}^{+}}$ & $\mathrm{Cl}^{-}$ & $\mathrm{K}^{+}$ & $\mathrm{H}_{2} \mathrm{O}$ \\
\hline$(9,9)$ & 42 & 33 & 19 & 26406 & 10 & 5 & 4 & 13765 \\
\hline$(8,8)$ & 13 & 4 & 3 & 18910 & 5 & 0 & 1 & 9468 \\
\hline$(7,7)$ & 1 & 0 & 0 & 9920 & 0 & 0 & 0 & 5008 \\
\hline
\end{tabular}

as ion sieves. That is, under some conditions, only particular type of ions will permeate whereas the others cannot. To investigate this possibility, we performed hydrostatic-pressure simulations in which a pressure difference was applied across the SWNT membranes with $\mathrm{Na}^{+}, \mathrm{K}^{+}$, and $\mathrm{Cl}^{-}$coexisting in the simulation. The number of each type of ion that passed across it was counted, as shown in Table 4. No ions passed through the $(5,5)$ or $(6,6)$ SWNT membranes in our simulations because of the large energy barriers they faced. However, as noted previously, ${ }^{25}$ water still passed across the membranes in these situations, highlighting their potential use for desalination. In the remaining situations, more $\mathrm{Na}^{+}$ions crossed the membrane than $\mathrm{K}^{+}$ions, which is consistent with the PMF results given that $\mathrm{K}^{+}$faces a higher barrier in these cases. Although the 1D PMF barrier difference between $\mathrm{Na}^{+}$and $\mathrm{K}^{+}$ in $(7,7)$ or $(8,8)$ SWNTs is not distinct, we can still see an obvious selectivity in the hydrostatic-pressure simulations. This is also in line with the 2D PMF results, where the differences in the barrier heights for $\mathrm{Na}^{+}$and $\mathrm{K}^{+}$in $(7,7)$ and $(8,8)$ SWNTs are clearer than for the corresponding 1D profiles as shown in Table 1. In some cases, more $\mathrm{Cl}^{-}$ions than $\mathrm{K}^{+}$ions passed; however, it must be kept in mind that there are twice as many $\mathrm{Cl}^{-}$ions in the system than each of the other ion types, so when comparing selectivities, these values should be halved. In addition, analysis of the trajectory shows that some of the cases in which $\mathrm{Cl}^{-}$ions cross the membrane, they are accompanying $\mathrm{Na}^{+}$ions as a result of attractive pair interactions. Moving in such "chaperone" pairs might assist in negating some of the energy costs associated with translocation.

Interestingly, no ions passed across the membrane composed of $(7,7)$ SWNTs under $100 \mathrm{MPa}$ pressure, only $\mathrm{Na}^{+}$ions passed through the $(7,7)$ SWNT membranes under $200 \mathrm{MPa}$, and both $\mathrm{Na}^{+}$and $\mathrm{K}^{+}$permeated through the $(8,8)$ SWNT under $100 \mathrm{MPa}$. These differences provide a possible way to discriminate different types of ions: Given a solution in which $\mathrm{Na}^{+}, \mathrm{Cl}^{-}$, and $\mathrm{K}^{+}$coexist, by applying $100 \mathrm{MPa}$ pressure across a $(8,8)$ SWNT membrane, one can discriminate $\mathrm{Cl}^{-}$from $\mathrm{Na}^{+}$and $\mathrm{K}^{+}$. Then, by applying $200 \mathrm{MPa}$ pressure across a $(7,7)$ SWNT membrane, one can separate $\mathrm{K}^{+}$from $\mathrm{Na}^{+}$. Of course, one could also obtain pure water by applying $100 \mathrm{MPa}$ or less across the $(7,7)$ SWNT membrane, given that water molecules can rapidly permeate in this case, as shown in the Table 4. Although the pressure needed is high in our simulation, it is possible to achieve similar results using much lower pressure by choosing membranes composed of wider SWNTs or charged or functionalized SWNTs, which can make the permeation barrier much smaller, ${ }^{11,14,26}$ or by waiting longer times. We note that such ion sieves would create large charge separations across the membranes that would act to prevent further ion movement. This problem could be avoided either by continuously flowing the solution on either side of the membrane or by using a mix of permeant and nonpermeant counterions.

These results shed further light on the hydrophobic gating mechanism proposed to operate in biological pores. As noted above, water permeates through all of the pores studied, but ions would not be expected to cross the three narrower pores without a very large driving force. Thus, a hydrophobically lined pore could be used to gate ions channels while still allowing a significant quantity of water molecules to cross. The water density inside pores has previously been used as an indicator of a hydrophobically gated pore. ${ }^{6}$ A low water density does suggest a large energy barrier to ions; however, it is still possible to find such a barrier even in cases with significant water density provided that the coordination number of ions in the pore is below the bulk value.

\section{Summary}

Using SWNTs as model hydrophobic pores, we have performed extensive MD simulations to investigate their intrinsic ion selectivity and its relation to pore size. The results indicate that narrow hydrophobic pores have intrinsic ion selectivity as supported both by free energy calculations and by simulations under hydrostatic pressure. The dehydration energy plays a dominant role in determining the selectivity because it has the largest role in determining the free energy barrier for ion entry in most cases. Using this reasoning, it is also possible to roughly describe the selectivity based on the coordination number in the pore, an explanation that shares some similarities with current explanations of selectivity in (hydrophilic) potassium channels. ${ }^{46,54}$ In the narrow pores, all ions have to be largely dehydrated, which impedes $\mathrm{Na}^{+}$most significantly because of its larger solvation energy. In the wider pores, however, $\mathrm{Na}^{+}$ can achieve a coordination number more similar to its bulk value than can the other ions, and thus, it is favored. In the widest pores, all ions gain close to their bulk coordination numbers, and the selectivity is relatively small. However, the FEP calculations show that the dehydration energy cannot explain the energy barrier to ion entry in all cases. The interaction between the ion or water and the pore becomes important in narrow pores, and the changing geometry of the solvation shell in the confined pores can alter the total energy barrier.

The ion selectivity of biological pores is usually associated with charged or polar groups that create favorable electrostatic environments or contacts for some types of ions but not others. As many biological channels, including the acetylcholine receptor and bacterial mechanosensitive channels, contain hydrophobic regions within their pores, we suspect that this will influence the selectivity of the pore in addition to any electrostatic effects from other regions of the protein. A continuous pore can also block the passage of ions ("hydrophobic gating") while still passing water. Our results also have interesting technological applications by suggesting a potential method to discriminate different types of ions. By carefully selecting the SWNT size and the pressure across the membrane, different types of ions can be extracted one by one. Therefore, carefully fabricated SWNT membranes can act as ion sieves, an application that we hope can be tested experimentally in the future.

Acknowledgment. This work was supported by funding from the National Health and Medical Research Council of Australia, an award under the merit allocation scheme on the APAC National Facility at the ANU, and additional computer time from iVEC.

Supporting Information Available: Results of test FEP simulations and full citation of ref 40 . This material is available free of charge via the Internet at http://pubs.acs.org.

\section{References and Notes}

(1) Unwin, N. J. Mol. Biol. 2005, 346, 967-989. 
(2) Chang, G.; Spencer, R.; Lee, A.; Barclay, M.; Rees, D. Science 1998, 282, 2220-2226. 1587.

(3) Bass, R.; Strop, P.; Barclay, M.; Rees, D. Science 2002, 298, 1582-

(4) Doyle, D. A.; Cabral, J. M.; Pfuetzner, R. A.; Kuo, A.; Gulbis, J. M.; Cohen, S. L.; Chait, B. T.; MacKinnon, R. Science 1998, 280, 6977.

(5) Jiang, Y.; Lee, A.; Chen, J.; Cadene, M.; Chait, B.; MacKinnon, R. Nature 2002, 417, 523-526.

(6) Beckstein, O.; Sansom, M. Phys. Biol. 2004, 1, 42-52.

(7) Beckstein, O.; Sansom, M. S. P. Phys. Biol. 2006, 3, 147-159.

(8) Corry, B. Biophys. J. 2006, 90, 799-810.

(9) Hub, J. S.; de Groot, B. L. Proc. Natl. Acad. Sci. U.S.A. 2008, 105, 1198-1203. 9280 .

(11) Sumikama, T.; Saito, S.; Ohmine, I. J. Phys. Chem. B 2006, 110 20671-20677.

(12) Peter, C.; Hummer, G. Biophys. J. 2005, 89, 2222-2234.

(13) Dehez, F.; Tarek, M.; Chipot, C. J. Phys. Chem. B 2007, 111, $10633-10635$.

(14) Leung, K.; Rempe, S. B.; Lorenz, C. D. Phys. Rev. Lett. 2006, 96, 095504

(15) Hwang, H.; Schatz, G. C.; Ratner, M. A. J. Phys. Chem. B 2006 $110,26448-26460$

(16) Zhu, F.; Schulten, K. Biophys. J. 2003, 85, 236-244.

(17) Iijima, S. Nature 1991, 354, 56-58.

(18) Hummer, G.; Rasaiah, J. C.; Noworyta, J. P. Nature 2001, 414, $188-190$.

(19) Striolo, A. Nano Lett. 2006, 6, 633-639.

(20) Kalra, A.; Garde, S.; Hummer, G. Proc. Natl. Acad. Sci. U.S.A 2003, 100, 10175-10180.

(21) Takaiwa, D.; Hatano, I.; Koga, K.; Tanaka, H. Proc. Natl. Acad. Sci. U.S.A. 2008, 105, 39-43.

(22) Meng, L.; Li, Q.; Shuai, Z. J. Chem. Phys. 2008, 128, 134703.

(23) Holt, J. K.; Noy, A.; Huser, T.; Eaglesham, D.; Bakajin, O. Nano Lett. 2004, 4, 2245-2250.

(24) Koga, K.; Gao, G. T.; Tanaka, H.; Zeng, X. C. Nature 2001, 412, 802-805.

(25) Corry, B. J. Phys. Chem. B 2008, 112, 1427-1434

(26) Huang, B.; Xia, Y.; Zhao, M.; Li, F.; Liu, X.; Ji, Y.; Song, C. J. Chem. Phys. 2005, 122, 084708

(27) Wan, R.; Li, J.; Lu, H.; Fang, H. J. Am. Chem. Soc. 2005, 127, $7166-7170$.

(28) Skoulidas, A. I.; Ackerman, D. M.; Johnson, J. K.; Sholl, D. S. Phys. Rev. Lett. 2002, 89, 185901.

(29) Dellago, C.; Naor, M. M.; Hummer, G. Phys. Rev. Lett. 2003, 90, 105902.
(30) Mann, D. J.; Halls, M. D. Phys, Rev. Lett. 2003, 90, 195503.

(31) Liu, H.; Murad, S.; Jameson, C. J. J. Chem. Phys. 2006, 125, 084713.

(32) Lu, D.; Li, Y.; Ravaioli, U.; Schulten, K. Phys. Rev. Lett. 2005, 95, 246801.

(33) Miller, S. A.; Young, V. Y.; Martin, C. R. J. Am. Chem. Soc. 2001, 123, 12335-12342.

(34) Siwy, Z.; Heins, E.; Harrell, C. C.; Kohli, P.; Martin, C. R. J. Am. Chem. Soc. 2004, 126, 10850-10851.

(35) Harrell, C. C.; Kohli, P.; Siwy, Z.; Martin, C. R. J. Am. Chem. Soc. 2004, 126, 15646-15647.

(36) Holt, J. K; Park, H. G.; Wang, Y.; Stadermann, M; Artyukhin, A. B.; Grigoropoulos, C. P.; Noy, A.; Bakajin, O. Science 2006, 312, 1034 1037.

(37) Hinds, B. J.; Chopra, N.; Rantell, T.; Andrews, R.; Gavalas, V.; Bachas, L. G. Science 2004, 303, 62-65.

(38) Liu, H.; Jameson, C. J.; Murad, S. Mol. Simul. 2008, 34, 169-175.

(39) Phillips, J. C.; Braun, R.; Wang, W.; Gumbart, J.; Tajkhorshid, E.; Villa, E.; Chipot, C.; Skeel, R. D.; Kale, L.; Schulten, K. J. Comput. Chem. 2005, 26, 1781-1802.

(40) MacKerell, A. D., Jr.; et al. J. Phys. Chem. B 1998, 102, 35863616, See the Supporting Information for the full reference.

(41) Torrie, G.; Valleau, J. Chem. Phys. Lett. 1974, 28, 578-581.

(42) Kumar, S.; Bouzida, D.; Swendsen, R. H.; Kollman, P. A.; Rosenberg, J. M. J. Comput. Chem. 1992, 13, 1011-1021.

(43) Roux, B. Comput. Phys. Commun. 1995, 91, 275-282.

(44) Grossfield Laboratory Lab Research Home Page: http://membrane. urmc.rochester.edu.

(45) Chipot, C.; Pearlman, D. Mol. Simul. 2002, 28, 1-12.

(46) Thomas, M.; Jayatilaka, D.; Corry, B. Biophys. J. 2007, 93, 26352643

(47) Gao, J.; Kuczera, K.; Tidor, B.; Karplus, M. Science 1989, 244, $1069-1072$

(48) Pearlman, D. J. Phys. Chem. 1994, 98, 1487-1493. 160 . 57.

50) Zhu, F.; Tajkhorshid, E.; Schulten, K. Biophys. J. 2004, 86, 50-

(51) Allen, T. W.; Andersen, O. S.; Roux, B. Biophys. Chem. 2006, $124,251-267$.

(52) Corry, B.; Chung, S. Cell. Mol. Life Sci. 2006, 63, 301-315.

(53) Tai, K.; Haider, S.; Grottesi, A.; Sansom, M. S. Eur. Biophys. J. 2009, 38, 347-354.

(54) Bostick, D.; Brooks, C. Proc. Natl. Acad. Sci. U.S.A. 2007, 104, 9260-9265.

JP810102U 